\title{
Balancing curability and unnecessary surgery in the context of computed tomography screening for lung cancer
}

\author{
Raja Flores, MD, ${ }^{\mathrm{a}}$ Thomas Bauer, MD, ${ }^{\mathrm{b}}$ Ralph Aye, MD ${ }^{\mathrm{c}}$ Shahriyour Andaz, MD, ${ }^{\mathrm{d}}$ Leslie Kohman, MD, \\ Barry Sheppard, MD, ${ }^{\mathrm{f}}$ William Mayfield, MD, ${ }^{\mathrm{g}}$ Richard Thurer, MD, ${ }^{\mathrm{h}}$ Michael Smith, MD, ${ }^{\mathrm{i}}$ \\ Robert Korst, MD, ${ }^{\mathrm{j}}$ Michaela Straznicka, MD ${ }^{\mathrm{k}}$ Fred Grannis, MD, ${ }^{1}$ Harvey Pass, MD, ${ }^{\mathrm{m}}$ \\ Cliff Connery, MD, ${ }^{a}$ Rowena Yip, MPH, ${ }^{a}$ James P. Smith, MD, ${ }^{\mathrm{n}}$ David Yankelevitz, MD, ${ }^{\mathrm{a}}$ \\ Claudia Henschke, PhD, MD, ${ }^{\mathrm{a}}$ and Nasser Altorki, MD, ${ }^{\circ}$ for the I-ELCAP Investigators
}

Objective: Surgical management is a critical component of computed tomography (CT) screening for lung cancer. We report the results for US sites in a large ongoing screening program, the International Early Lung Cancer Action Program (I-ELCAP).

Methods: We identified all patients who underwent surgical resection. We compared the results before (1993-2005) and after (2006-2011) termination of the National Lung Screening Trial to identify emerging trends.

Results: Among 31,646 baseline and 37,861 annual repeat CT screenings, 492 patients underwent surgical resection; 437 (89\%) were diagnosed with lung cancer; 396 (91\%) had clinical stage I disease. In the 54 $(11 \%)$ patients with nonmalignant disease, resection was sublobar in 48 and lobectomy in 6 . The estimated cure rate based on the 15-year Kaplan-Meier survival for all 428 patients (excluding 9 typical carcinoids) with lung cancer was $84 \%$ (95\% confidence interval [CI], 80\%-88\%) and $88 \%$ (95\% CI, 83\%-92\%) for clinical stage I disease resected within 1 month of diagnosis. Video-assisted thoracoscopic surgery and sublobar resection increased significantly, from $10 \%$ to $34 \%(P<.0001)$ and $22 \%$ to $34 \%(P=.01)$ respectively; there were no significant differences in the percentage of malignant diagnoses $(90 \% \mathrm{vs} 87 \%, P=.36)$, clinical stage I $(92 \%$ vs $89 \%, P=.33)$, pathologic stage $\mathrm{I}(85 \%$ vs $82 \%, P=.44)$, tumor size $(P=.61)$, or cell type $(P=.81)$.

Conclusions: The frequency and extent of surgery for nonmalignant disease can be minimized in a CT screening program and provide a high cure rate for those diagnosed with lung cancer and undergoing surgical resection. (J Thorac Cardiovasc Surg 2014;147:1619-26)

Earn CME credits at

http://jtcvs.com/cme/home

Computed tomography (CT) screening for lung cancer has been shown to lead to earlier diagnosis and high cure rates of lung cancer in the United States ${ }^{1,2}$ and Japan. ${ }^{3,4}$ In 2011, the National Lung Screening Trial (NLST) ${ }^{5}$ confirmed that CT screening reduces mortality from lung cancer to a

\footnotetext{
From the Department of Surgery and Radiology, ${ }^{\mathrm{a}}$ Icahn School of Medicine at Mout Sinai, New York, NY; Department of Surgery, ${ }^{\mathrm{b}}$ Christiana Care, Helen F. Graham Cancer Center, Newark, Del; Department of Surgery, ${ }^{\mathrm{c}}$ Swedish Medical Center, Seattle, Wash; Department of Surgery, ${ }^{\mathrm{d}}$ South Nassau Communities Hospital,

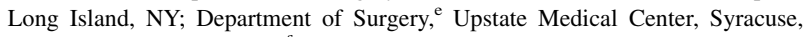
NY; Department of Surgery, ${ }^{\mathrm{f}}$ Mills-Peninsula Health Services, San Mateo, Calif; Department of Surgery, ${ }^{\mathrm{g}}$ Wellstar Health System, Marietta, Ga; Department of Surgery, ${ }^{\mathrm{h}}$ Jackson Memorial Hospital, University of Miami, Miami, Fla; Department of Surgery, ${ }^{\mathrm{i}}$ Georgia Institute for Lung Cancer Research, Atlanta, Ga; Department of Surgery, ${ }^{\mathrm{j}}$ The Valley Hospital Cancer Center, Paramus, NJ; Department of Surgery, ${ }^{\mathrm{k}}$ John Muir Cancer Institute, Concord, Calif; Department of Surgery, ${ }^{\mathrm{l}}$ City of Hope National Medical Center, Duarte, Calif; Department of Surgery, ${ }^{\mathrm{m}}$ New York University Medical Center, New York, NY; Department of Medicine, ${ }^{\mathrm{n}}$ Weill Cornell Medical College, New York, NY; and Department of Cardiovascular Surgery, ${ }^{\circ}$ Weill Cornell Medical College, New York, NY.

Disclosures: David Yankelevitz is a named inventor on several patents and patent applications relating to the evaluation of diseases of the chest including measurement of nodules. Some of these, which are owned by Cornell Research
}

sufficient extent to recommend screening for high-risk people, and CT screening is being provided as part of clinical care throughout the United States. In any screening program, a balance needs to be maintained between the benefits and potential harms of the screening, and this requires a well-designed screening protocol that specifies the indications for diagnostic tests as well as for surgical interventions.

We wanted to examine the surgical results and management of lung cancer in the United States in a large ongoing

Foundation (CRF), are nonexclusively licensed to General Electric. As an inventor of these patents, Dr Yankelevitz is entitled to a share of any compensation that CRF may receive from its commercialization of these patents. Claudia Henschke is also an inventor of the patents and pending patents owned by CRF. Since April 2009, she has divested herself of all royalties and other interests arising from these Fred Grannis has provided paid written expert witness and oral deposition testimony in 3 state medical monitoring lawsuits against Philip Morris Corporation between 2007 and the present date. All other authors have nothing to disclose with regard to commercial support.

The I-ELCAP Investigators are listed in Appendix 1.

Received for publication April 23, 2013; revisions received Aug 8, 2013; accepted for publication Nov 1, 2013; available ahead of print Dec 11, 2013

Address for reprints: Claudia Henschke, PhD, MD, Department of Radiology,

Mount Sinai School of Medicine, 1 Gustave Levy Place, New York, NY 10029

(E-mail: claudia.henschke@mountsinai.org).

0022-5223/\$36.00

Copyright $(C) 2014$ by The American Association for Thoracic Surgery

http://dx.doi.org/10.1016/j.jtcvs.2013.11.001 


\section{Abbreviations and Acronyms \\ $\mathrm{CI}=$ confidence interval \\ CT $=$ computed tomography \\ I-ELCAP $=$ International Early Lung Cancer Action Program \\ NLST = National Lung Screening Trial \\ PET = positron emission tomography \\ VATS $=$ video-assisted thoracoscopic surgery}

screening program and focus on differences since 2006, when screening ended in the NLST, to identify evolving trends. We reviewed all surgical interventions at institutions in the United States since 1993 within the International Early Lung Cancer Action Program (I-ELCAP), which provides a common protocol. We also wanted to assess the frequency and magnitude of surgery for nonmalignant disease.

\section{METHODS}

This report draws from the database of our International Early Lung Cancer Action Program (I-ELCAP), in which CT screening for lung cancer was performed according to a common protocol. ${ }^{6}$ The common protocol specified the initial CT test, the definition of positive results, and the recommended diagnostic work-up. The protocol required that all subjects be asymptomatic and deemed by their physician as a suitable candidate for surgical resection if a diagnosis of lung cancer was made, but the enrollment criteria with regard to age and smoking status was decided by each participating institution. Although the protocol makes recommendations for the work-up of positive results, the decision on how to proceed was left to each participant and the referring physician, and the actual diagnostics were documented. The type and extent of surgery was not mandated by the protocol but was according to the standard of care at each institution. Consent was obtained from all participants according to protocols compliant with the Health Insurance Portability and Accountability Act and approved by the Institutional Review Boards of the collaborating institutions. The collaborating institutions are in large metropolitan areas, major academic centers, and smaller communitybased medical centers. The participants had a history of smoking or exposure to airborne carcinogens from their occupation or from exposure to secondhand smoke; their ages ranged from 40 to 85 years.

This report focuses on the screening sites in the United States. For each of these sites, we identified all instances of thoracic surgery for first primary lung cancer prompted by results of the low-dose CT screening, which was guided by the I-ELCAP screening regimen; the decision on how to proceed was left to each participant and the referring physician and was documented in the management system. ${ }^{6}$ The tumor size and clinical stage were based on the results of CT scans (and positron emission tomography [PET], if done) that were performed closest in time to the recommendation for biopsy of a nodule. The surgery, outcome, and follow-up were documented by the principal investigator at each participating institution. This included reporting of all postoperative deaths occurring within 30 days of the surgery regardless of the cause of death. In all instances of a nonmalignant diagnosis, the final histologic diagnosis was obtained. Staging classification for lung cancer was made centrally based on the American Joint Committee on Cancer Cancer Staging Manual, sixth edition, with 1 exception. Cases of multiple adenocarcinomas $(<30 \mathrm{~mm}$ in diameter) without lymph node metastases were classified as synchronous primaries and considered to be stage $\mathrm{I}^{6,7}$ Patients who underwent bronchoscopy, endobronchial ultrasound biopsy, mediastinoscopy, scalene node biopsy, and transthoracic fine needle aspiration only and had no subsequent surgical procedure were excluded from the final surgical analysis. In case of known death, the date and cause were obtained from the patient's physician and/or family members. Any death resulting from treatment was considered a lung cancer death. Follow-up time from diagnosis onward to death from lung cancer, last contact, or April 30, 2013, whichever came first, was calculated for each case. The follow-up time ranged from 1 to 209 months (median 72 months).

We constructed Kaplan-Meier curves for lung cancer-specific survival on the date of diagnosis, inherently conditional on not dying of any other cause, in all cases of resection, and for those diagnosed in clinical stage I who underwent resection within 1 month of diagnosis. We also constructed survival curves for patients who underwent resection, regardless of the timing, and were found to have pathologic stage I disease by tumor diameter of the pathology specimen. From these long-term survival Kaplan-Meier lung cancer-specific survival curves, we used the asymptotic rates as our estimate of the cure rate. Typical carcinoids were excluded from all Kaplan-Meier survival analyses.

Comparisons of the patient and surgical characteristics of the 2 time intervals (1993-2005 and 2006-2011) were made using $\chi^{2}$ test or the Fisher exact test. All statistical analyses were performed using SAS version 9.2 (SAS Institute, Inc, Cary, NC). Frequencies and percentages and the corresponding $P$ values were calculated.

\section{RESULTS}

Between 1993 and 2011, 31,646 baseline and 37,861 annual repeat screenings were performed at US sites. These screenings resulted in 492 patients undergoing surgical resection, of which $437(89 \%)$ were diagnosed with lung cancer (Table 1). Postsurgical death occurred in $3(0.6 \%)$ patient; no deaths occurred in patients with a nonmalignant diagnosis or those undergoing video-assisted thoracoscopic surgery (VATS).

Among the 437 patients diagnosed with lung cancer, 396 $(91 \%)$ had clinical stage I disease and after surgery, 368 $(84 \%)$ had pathologic stage I disease. The cure rate for all 428 (excluding 9 typical carcinoids) patients diagnosed with lung cancer, as estimated by the asymptote of the Kaplan-Meier lung cancer-specific survival analysis, was $84 \% \quad(95 \%$ confidence interval [CI], 80\%-88\%) (Figure 1). For the 273 patients diagnosed with clinical stage I lung cancer and who underwent resection within 1 month of diagnosis, the estimated cure rate was $88 \%$ (95\% CI, 83\%-92\%).

The estimated cure rate for patients with pathologic stage I lung cancer was $95 \%(95 \% \mathrm{CI}, 90 \%-99 \%)$ for cancers $10 \mathrm{~mm}$ or less in diameter $(\mathrm{n}=106), 87 \%(95 \% \mathrm{CI}$, $81 \%-93 \%)$ for cancers $11-20 \mathrm{~mm}(\mathrm{n}=180)$, and $83 \%$ $(95 \%$ CI, $72 \%-93 \%)$ for cancers $21-30 \mathrm{~mm}(\mathrm{n}=60)$ (Figure 2).

Of the 492 surgical resections, $90(18 \%)$ were performed by VATS and the remaining $402(82 \%)$ by thoracotomy. Of these 492 surgical procedures, 230 had a preoperative diagnosis of lung cancer, which was confirmed for 228 $(99 \%)$ patients; $2(1 \%)$ were not malignant when resected. The remaining 262 patients did not have a preoperative diagnosis and of them, $209(80 \%)$ were diagnosed with lung cancer, 1 with renal cell carcinoma, and $52(20 \%)$ 
TABLE 1. Characteristics of all I-ELCAP patients undergoing surgery in institutions in the United States

\begin{tabular}{lcccc}
\hline & $\begin{array}{c}\text { Total } \\
(\mathbf{N}=\mathbf{4 9 2})\end{array}$ & $\begin{array}{c}\mathbf{1 9 9 3 - 2 0 0 5} \\
(\mathbf{n}=\mathbf{3 2 9})\end{array}$ & $\begin{array}{c}\mathbf{2 0 0 6}-2011 \\
(\mathbf{n}=\mathbf{1 6 3})\end{array}$ & $\begin{array}{c}\boldsymbol{P} \\
\text { value }\end{array}$ \\
\hline Median age, y & 65 & 66 & 64 & .0008 \\
Male, n (\%) & $210(43)$ & $146(44)$ & $64(39)$ & .28 \\
White, n (\%) & $460(93)$ & $307(93)$ & $153(94)$ & .82 \\
Lesion location, n (\%) & & & & \\
RUL & $182(37)$ & $120(36)$ & $62(38)$ & .67 \\
RML & $23(5)$ & $16(5)$ & $7(4)$ & \\
RLL & $90(18)$ & $56(17)$ & $34(21)$ & \\
LUL & $116(24)$ & $78(24)$ & $38(23)$ & \\
LLL & $81(16)$ & $59(18)$ & $22(13)$ & \\
Lesion size on CT, n (\%) & & & & \\
$\quad<5$ mm & $9(2)$ & $5(2)$ & $4(2)$ & .88 \\
5-9 mm & $97(20)$ & $64(19)$ & $33(20)$ & \\
10-14 mm & $178(36)$ & $121(37)$ & $57(35)$ & \\
$\geq 15$ mm & $204(41)$ & $137(42)$ & $67(41)$ & \\
Patchy/unmeasurable size & $4(1)$ & $2(1)$ & $2(1)$ & \\
Preoperative PET scan, n (\%) & $217(44)$ & $108(33)$ & $109(67)$ & $<.0001$ \\
Preoperative diagnosis n (\%) & $230(47)$ & $173(53)$ & $57(35)$ & .0002 \\
Methods of resection, n (\%) & & & & \\
$\quad$ VATS & $90(18)$ & $34(10)$ & $56(34)$ & $<.0001$ \\
$\quad$ Thoracotomy & $402(82)$ & $295(90)$ & $107(66)$ & \\
Final diagnosis, n (\%) & & & & \\
Lung cancer & $437(89)$ & $296(90)$ & $141(87)$ & .36 \\
Renal cell carcinoma & 1 & 1 & 0 & \\
Nonmalignant & $54(11)$ & $32(10)$ & $22(10)$ & \\
\hline
\end{tabular}

I-ELCAP, International Early Lung Cancer Action Program; RUL, right upper lobe; $R M L$, right middle lobe; $R L L$, right lower lobe; $L U L$, left upper lobe; $L L L$, left lower lobe; $C T$, computed tomography; PET, positron emission tomography; VATS, videoassisted thoracoscopic surgery.

with a nonmalignant diagnosis. The percentage of malignant diagnoses among those who had a preoperative biopsy was significantly higher than in those who did not have a preoperative diagnosis $(99 \%$ vs $80 \%, P<.0001)$. Median time to diagnosis was 4 months (interquartile range [IQR] 1.8-14.6) and median time between diagnosis and surgery was 0.0 months (IQR 0.0-1.2).

Among the 437 patients diagnosed with lung cancer, $81(18 \%)$ were treated by sublobar resection (wedge or anatomic segmentectomy), $352(81 \%)$ by lobectomy or bilobectomy, and only $4(1 \%)$ by pneumonectomy (Table 2). Adenocarcinoma was the most frequent tumor histology, followed by squamous cell, large cell, and small cell tumors (Table 2).

Among the 54 patients who had a nonmalignant diagnosis, most (46) underwent wedge resection, 2 had anatomic segmentectomy, and 6 had a lobectomy (Table 3). Detailed review of the histology of these 54 nonmalignant cases revealed chronic inflammation/fibrosis (21), granuloma (15), pneumonia requiring treatment (7), hamartoma (5), amyloidosis (2), atypical cells suggestive but not diagnostic of adenocarcinoma (2), lipoma (1), and lymph node (1).
Among these 54 patients with a nonmalignant diagnosis at surgery, 8 had a nodule diameter of $15 \mathrm{~mm}$ or more and for them, the protocol recommends additional options of an immediate PET scan or biopsy. Among these 8, none demonstrated growth but 6 had a positive PET scan. The remaining 46 patients had nodules less than $15 \mathrm{~mm}$ in diameter, and for them growth assessment was recommended. However, only 2 demonstrated growth at a malignant rate and both had PET scans with indeterminate uptake; the remaining 44 did not show growth (16 had PET scans: 3 were positive, 4 indeterminate, and 9 negative). Thus, based on the I-ELCAP protocol recommendations, only 6 of the larger nodules $(\geq 15 \mathrm{~mm})$ and 2 of the smaller nodules $(<15 \mathrm{~mm})$ would have been recommended for surgery and thus the number of patients undergoing surgical resection for nonmalignant disease would have been reduced from 54 to 8 and the number of surgical resections would have been reduced to 445 , which would have yielded a malignancy rate of $98 \%(437 / 445)$.

\section{Comparison of 2 Time Periods (1993-2005 vs 2006-2011)}

There was no significant difference in the percentage of malignant diagnoses among all resections $(90 \%$ vs $87 \%$, $P=.36$ ) when comparing the 2 time periods (Table 1). However, the percentage of patients who had a preoperative nonsurgical biopsy diagnosis of lung cancer significantly decreased from the first time period to the second $(53 \%$ vs $35 \%, P=.0002)$; and the use of PET scans increased significantly $(33 \%$ vs $67 \%, P<.0001)$.

The frequency of VATS resection $(10 \%$ vs $34 \%$, $P<.0001)$ increased from the first to the second time period for all resections (Table 1) as well as for lung cancer resections $(6 \%$ vs $33 \%, P<.0001)$ (Table 2$)$. Similarly, the frequency of sublobar resections (22\% vs $34 \%$, $P=.01)$ increased overall and among cancer resections ( $16 \%$ vs $25 \%, P=.02$ ) (Table 2$)$. Surgical mortality was lower in the second time period $(0.9 \%$ [ 3 of 329] vs $0 \%$ [0 of 163], $P=.22$ ), although not significantly.

The distribution by tumor size $(P=.61)$ and by cell type $(P=.81)$ was essentially the same for each of the 2 time periods (Table 2). Similarly, the percentages of patients diagnosed with clinical stage I disease $(92 \%$ vs $89 \%$, $P=.33)$ and pathologic stage I disease $(85 \%$ vs $82 \%$, $P=.44)$ were essentially the same.

\section{DISCUSSION}

In clinical practice, in the absence of screening, significant advances have changed the management of patients with lung cancer over the past several decades. Before CT screening, 10-year survival of $80 \%$ for pathologic stage I lung cancer (diameter $\leq 20 \mathrm{~mm}$ ) ${ }^{8}$ and $93 \%$ for pathologic stage I lung cancer (diameter $<10 \mathrm{~mm}$ ) had been demonstrated. Since then, CT scans have replaced 


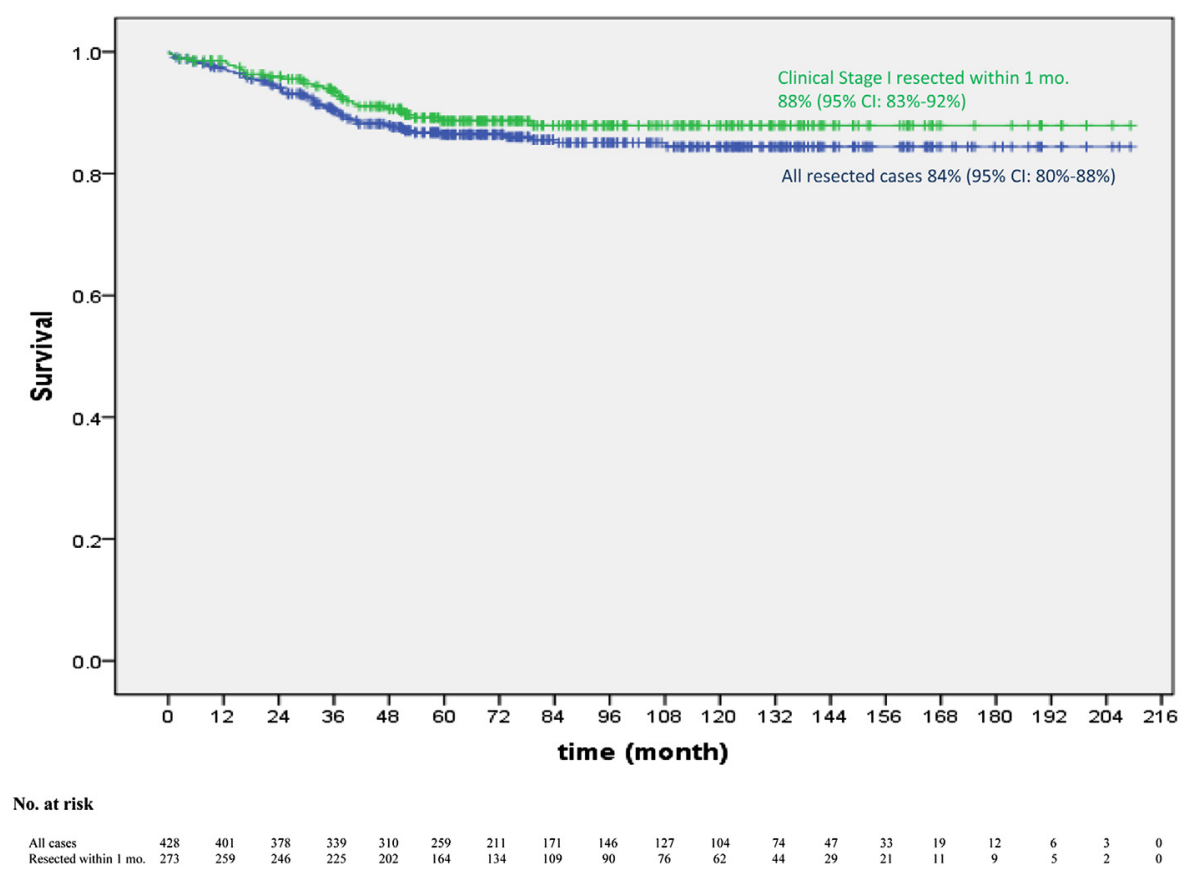

FIGURE 1. Kaplan-Meier lung cancer-specific survival for all patients who underwent resection, separately for all stages and for all clinical stage I cases resected within 1 month of diagnosis. Typical carcinoids were excluded. $C I$, Confidence interval.

chest radiographs as the imaging gold standard and this has increased the number of small lung cancers that have been detected..$^{2-5}$ Also, VATS has emerged as an alternative therapeutic approach to thoracotomy. There is now a general consensus that, based on comparative studies, VATS is the preferred approach because it is associated with a significant reduction in perioperative morbidity than pulmonary resection by thoracotomy. ${ }^{10}$

Ideally, surgery for lung cancer should always be preceded by a nonsurgical biopsy, which establishes the diagnosis, the cell type, and other relevant tumor characteristics, so that the extent of the resection can be planned

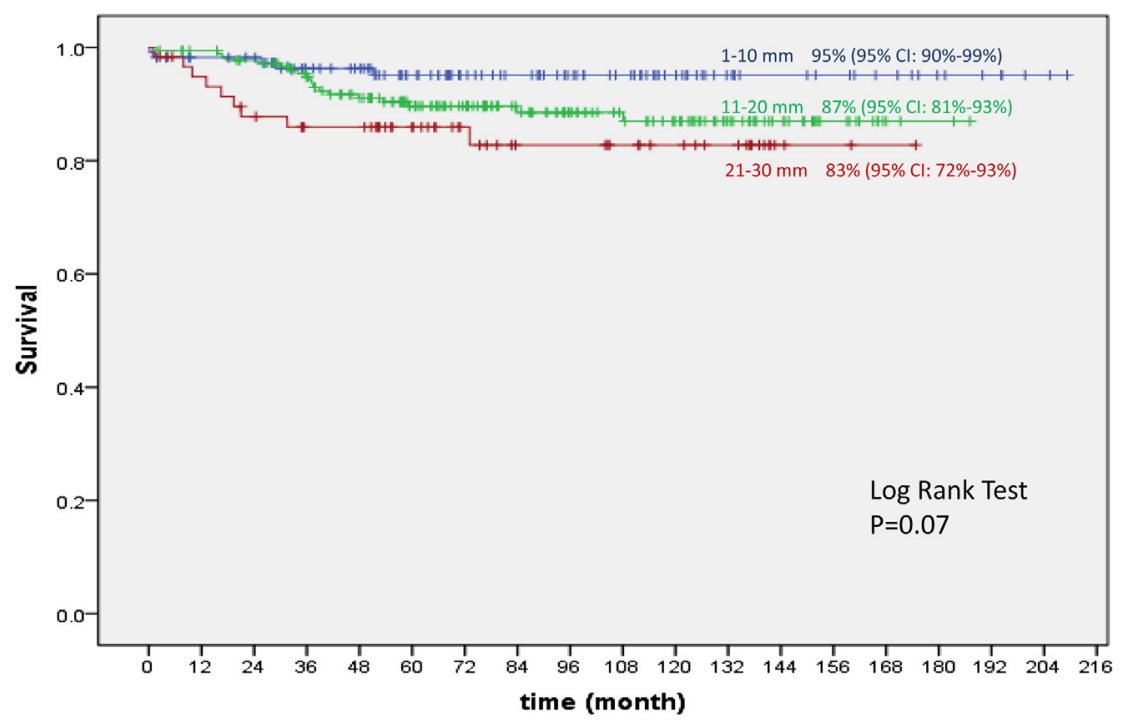

No. at risk

$\begin{array}{lccccccccccccccccccc}1-10 \mathrm{~mm} & 106 & 100 & 99 & 86 & 81 & 67 & 55 & 50 & 42 & 36 & 27 & 19 & 14 & 12 & 11 & 7 & 5 & 2 & 0 \\ 11-20 \mathrm{~mm} & 180 & 175 & 168 & 157 & 141 & 119 & 102 & 80 & 66 & 57 & 48 & 33 & 22 & 12 & 4 & 2 & 0 & 0 & 0 \\ 21-30 \mathrm{~mm} & 60 & 54 & 49 & 44 & 44 & 35 & 27 & 21 & 21 & 18 & 15 & 12 & 3 & 2 & 1 & 0 & 0 & 0 & 0\end{array}$

FIGURE 2. Kaplan-Meier lung cancer-specific survival curve for all patients diagnosed as pathologic stage I by tumor diameter of the pathology specimen. Typical carcinoids were excluded. $C I$, Confidence interval. 
TABLE 2. Extent and type of surgery for patients with a lung cancer diagnosis

\begin{tabular}{|c|c|c|c|c|}
\hline & Total $(N=437)$ & $1993-2005(n=296)$ & $2006-2011(n=141)$ & $P$ value \\
\hline \multicolumn{5}{|l|}{ Type of resection, $\mathrm{n}(\%)$} \\
\hline \multicolumn{5}{|l|}{ Sublobar } \\
\hline Wedge & $53(12)$ & $30(10)$ & $23(16)$ & .08 \\
\hline Segmentectomy & $28(6)$ & $16(5)$ & $12(9)$ & \\
\hline Lobectomy & $340(78)$ & $236(80)$ & $104(74)$ & \\
\hline Bilobectomy & $12(3)$ & $11(4)$ & $1(1)$ & \\
\hline Pneumonectomy & $4(1)$ & $3(1)$ & $1(1)$ & \\
\hline \multicolumn{5}{|l|}{ Method of resection } \\
\hline VATS & $65(15 \%)$ & $19(6 \%)$ & $46(33 \%)$ & $<.0001$ \\
\hline Thoracotomy & $372(85 \%)$ & $277(94 \%)$ & $95(67 \%)$ & \\
\hline \multicolumn{5}{|l|}{ Cell type } \\
\hline Adeno & $315(72 \%)$ & $210(71 \%)$ & $105(74 \%)$ & .81 \\
\hline Squamous & $65(15 \%)$ & $46(16 \%)$ & $19(13 \%)$ & \\
\hline Large cell & $26(6)$ & $19(6)$ & $7(5)$ & \\
\hline Small cell & $16(4)$ & $12(4)$ & $4(3)$ & \\
\hline Other* & $15(3)$ & $9(3)$ & $6(4)$ & \\
\hline Median tumor diameter, $\mathrm{mm}$ (IQR) & $13.0(10.0-20.0)$ & $13.5(10.0-20.0)$ & $13.0(10.0-21.0)$ & .61 \\
\hline \multicolumn{5}{|l|}{ Stage I, n (\%) } \\
\hline Clinical & $396(91)$ & $271(92)$ & $125(89)$ & .33 \\
\hline Pathologic & $368(84)$ & $252(85)$ & $116(82)$ & .44 \\
\hline
\end{tabular}

VATS, Video-assisted thoracoscopic surgery; IQR, interquartile range. *Nine typical carcinoids are included, 7 in 1993-2005 and 2 in 2006-2011.

preoperatively and surgery for benign disease is markedly reduced. Although ideal, this is not always feasible and when surgical biopsy is warranted, VATS can be performed in most cases with a hospital stay of less than 24 hours. ${ }^{11-13}$ Furthermore, in the context of CT screening for lung cancer, further work-up of nodules identified as a result of the screening is directed by protocol. Thus, the CT findings, particularly of small nodules, can be managed appropriately by follow-up imaging and can often be diagnosed by nonsurgical methods such as fine needle aspiration and navigational bronchoscopy after demonstration of nodule growth by two-dimensional or volumetric methods. ${ }^{14-17}$

The conventional approach for undiagnosed lung nodules has usually emphasized surgical resection because of the high mortality rate of untreated lung cancer. However,

TABLE 3. Extent of surgery performed on patients who had a benign diagnosis

\begin{tabular}{lcccc}
\hline & $\begin{array}{c}\text { Total } \\
(\mathbf{N}=\mathbf{5 4})\end{array}$ & $\begin{array}{c}\mathbf{1 9 9 3 - 2 0 0 5} \\
(\mathbf{n = 3 2})\end{array}$ & $\begin{array}{c}\mathbf{2 0 0 6 - 2 0 1 1} \\
(\mathbf{n = 2 2 )}\end{array}$ & $\begin{array}{c}\boldsymbol{P} \\
\text { value }\end{array}$ \\
\hline Type of resection, n (\%) & & & & \\
$\quad$ Sublobar & & & & \\
$\quad$ Wedge & $46(85)$ & $27(84)$ & $19(86)$ & 1.0 \\
$\quad$ Segmentectomy & $2(4)$ & $1(3)$ & $1(5)$ & \\
Lobectomy & $6(11)$ & $4(13)$ & $2(9)$ & \\
Bilobectomy & $0(0)$ & $0(0)$ & $0(0)$ & \\
Pneumonectomy & $0(0)$ & $0(0)$ & $0(0)$ & \\
Method of resection, n (\%) & & & & \\
$\quad$ VATS & $25(46)$ & $15(47)$ & $10(45)$ & .92 \\
Thoracotomy & $29(54)$ & $17(53)$ & $12(55)$ & \\
\hline
\end{tabular}

VATS, Video-assisted thoracoscopic surgery. this practice results in a high rate of surgical resection of benign lung lesions. The literature demonstrates that the rate of benign diagnosis is $40-80 \%$ and as low as $9 \%$ when surgical resection is performed for solitary pulmonary nodules. ${ }^{18-21}$ There is no accepted standard negative rate of diagnostic surgical intervention for suspected malignant disease. The best available data comes from a randomized clinical trial reported in 1995 by the Lung Cancer Study Group, which established lobectomy as the gold standard for lung cancer. It demonstrated a surgical resection rate for benign lesions of $24 \%{ }^{22}$ The NLST recently reported a rate of $21 \%$ for surgery not resulting in a diagnosis of lung cancer. ${ }^{5}$ The I-ELCAP data reported here demonstrate a rate of surgical intervention of $11 \%$ (54 of 492) for benign disease, a major improvement in the rates previously reported. Moreover, if the CT screening protocol had been followed as recommended, the benign resection rate would have been less than $2 \%$ and no lung cancer cases would have been missed. Starnes and colleagues ${ }^{23}$ demonstrated the plausibility of a practice that minimizes surgical intervention for benign disease without missing curable lung cancers by using a multidisciplinary team approach even in geographic regions where the finding of pulmonary nodules is extremely common because of endemic histoplasmosis. The recommendation of the International Association for the Study of Lung Cancer surgical panel indicates that $85 \%$ of surgical diagnoses should result in a malignant diagnosis. ${ }^{24}$ It seems clear that a balance must be attained that minimizes surgical intervention for benign disease while maximizing curative surgery for lung cancer. 
The advent of VATS has greatly reduced the morbidity of lung surgery. Its use for the diagnosis and treatment of lung cancer has led to fewer complications, less pain, lower cost, and shorter length of hospital stay compared with thoracotomy. ${ }^{11-13}$ The Society of Thoracic Surgeons' database has demonstrated a 4-fold increase in the use of VATS since 2006 and anticipates more widespread use in the future with better surgeon education. ${ }^{23}$ The experience with VATS wedge resection for the diagnosis of benign disease in the current literature demonstrates a mortality rate of zero. ${ }^{18-21}$ This is reflected in both the I-ELCAP and NLST data. The current I-ELCAP data demonstrate an increase from $10 \%$ to $34 \%$ in the use of VATS technology over the 2 time periods, reflecting its greater use in the period after the termination of the NLST. These data also show that following a well-defined protocol that requires demonstration of nodule growth and/or nonsurgical biopsy can markedly reduce the frequency of resecting a nonmalignant nodule. Therefore, all CT screening programs should include thoracic surgeons well versed in performing VATS resections in their multidisciplinary review panels and conferences to minimize morbidity in their screening program.

Overall, surgical morbidity is determined not only by the rate of resection for benign disease but also the extent of the surgery. Diagnostic lobectomies for benign disease pose the greatest potential morbidity to patients enrolled in a lung screening program. This situation must be avoided as screening is adopted more universally and should be reserved as last resort after all nonsurgical and minimally invasive diagnostic modalities have been exhausted. This constitutes a paradigm shift to be embraced by thoracic surgeons who normally have a low threshold for performing lobectomy for definitive diagnosis and treatment of lesions suspicious of lung cancer in patients with adequate pulmonary function. Our data have demonstrated that among the 492 patients who underwent surgery, only $1 \%$ had lobectomy for benign disease compared with the best data available from experienced centers that report benign lobectomy rates of $5 \%$ to $15 \%$. $^{21,22}$

The recent recommendation by the United States Preventative Services Task Force to screen annually underscores the need for careful surgical selection to maximize effectiveness. ${ }^{26}$ Surgery for benign lesions, especially by lobectomy, should be minimized.

There are currently also proponents of less extensive surgery, such as wedge and segmentectomy, on patients with lung cancer for definitive treatment as well as for cases of possible benign disease. CT scans can now identify lesions at such a small size that the question of lobar versus sublobar resection has resurfaced as to their level of equipoise. The randomized controlled trial initiated by the Cancer and Leukemia Group B is currently well underway to address this question for lung cancers of $2 \mathrm{~cm}$ or less in diameter. Our study demonstrates a low rate of stage migration in the CT-screened population, which would support sublobar resection for lung cancer, and the data demonstrated a statistically significant $10 \%$ increase in the number of sublobar resections in the most recent time period (2006-2011).

Despite the ongoing debate about the optimal surgical procedure, it seems clear from the I-ELCAP data that the treatment of smaller, earlier stage lung cancer has the capability to result in higher lung preservation, excellent operative outcomes, and curability. These findings confirm the work of Pastorino and colleagues ${ }^{27}$ regarding surgical mortality, which demonstrated that resection of early-stage lung cancers had a favorable effect on surgical mortality, not only by preventing the need for pneumonectomy but by also reducing mortality after lobectomy. Our data demonstrate a pneumonectomy rate of only $1 \%$ compared with $7 \%$ and $14 \%$ as reported by the Society of Thoracic Surgeons and the American College of Surgeons, respectively. ${ }^{22}$ The postoperative surgical mortality in the $\mathrm{NLST}^{5}$ was $1 \%$, which is similar to the $0.6 \%$ demonstrated here. These findings translate into a reduction in surgical mortality, especially with the decrease in the frequency of pneumonectomy, which is associated with a mortality rate of $5 \%$ to $15 \% .^{25,28}$

In conclusion, unnecessary surgery for benign disease and the extent of lung resection in a CT-screened population can be minimized. When follow-up imaging over time to assess nodule growth and nonsurgical biopsy fail to provide a diagnosis, surgical biopsy by VATS wedge resection should be the procedure of choice. A diagnostic lobectomy should be required infrequently in an efficient CT screening program but when necessary as a procedure of last resort. In addition, CT screening can lead to a lower rate of pneumonectomy and improved operative outcomes. In lung cancer, a disease for which nonsurgical treatments have disappointing cure rates, screening leading to diagnosis at an early stage, followed by judicious use of surgery, can significantly improve cure rates and limit morbidity.

The screenings in the I-ELCAP pooled database have been supported in part by National Institutes of Health R01-CA-633931 and R01-CA-78905; Department of Energy DE-FG02-96SF21260; City of New York, Department of Health and Mental Hygiene; New York State Office of Science, Technology and Academic Research (NYSTAR); American Cancer Society; Starr Foundation; New York Community Trust; Rogers Family Fund; Foundation for Lung Cancer: Early Detection, Prevention, and Treatment (primary source from an unrestricted gift in 2000-2003 from the Vector Group, the parent company of Liggett Tobacco); Dorothy R. Cohen Foundation, Jacob and Malka Goldfarb Charitable Foundation; Auen/Berger Foundation; Berger Foundation; Mills-Peninsula Hospital Foundation, Tenet Healthcare Foundation; Ernest E. Stempel Foundation; Academic 
Medical Development Corporation; Columbia University Medical Center; Empire Blue Cross and Blue Shield; Eastman-Kodak Corporation; General Electric Corporation; Weill Medical College of Cornell University; Cornell University; New York Presbyterian Hospital; Swedish Hospital; Christiana Care Helen F. Graham Cancer Center; Holy Cross Hospital; Eisenhower Hospital; Jackson Memorial Hospital Health System; Evanston Northwestern Healthcare.

\section{References}

1. Henschke CI, McCauley DI, Yankelevitz DF, Naidich DP, McGuinness G, Miettinen OS, et al. Early Lung Cancer Action Project: overall design and findings from baseline screening. Lancet. 1999;354:99-105.

2. Henschke CI, Yankelevitz DF, Libby DM, Pasmantier MW, Smith JP, Miettinen OS. Survival of patients with stage I lung cancer detected on CT screening. N Engl J Med. 2006;355:1763-71.

3. Sobue T, Moriyama N, Kaneko M, Kusumoto M, Kobayashi T, Tsuchiya R, et al. Screening for lung cancer with low-dose helical computed tomography: anti-lung cancer association project. J Clin Oncol. 2002;20:911-20.

4. Sone S, Nakayama T, Honda T, Tsushima K, Li F, Haniuda M, et al. Long-term follow-up of a mass screening programme for lung cancer using mobile low-dose spiral computed tomography scanner. Lung Cancer. 2007;58:329-41.

5. The National Lung Screening Trial Research Team. Reduced lung-cancer mortality with low-dose computed tomographic screening. $N$ Engl J Med. 2011:365:395-409.

6. I-ELCAP protocol. http://www.ielcap.org/sites/default/files/ielcap.pdf. Accessed August 1, 2013.

7. Vazquez M, Carter D, Brambilla E, Gazdar A, Noguchi M, Travis WD, et al. Solitary and multiple resected adenocarcinomas after CT screening for lung cancer: histopathologic features and their prognostic implications. Lung Cancer. 2009;64:148-54.

8. Buell PE. The importance of tumor size in prognosis for resected bronchogenic carcinoma. J Surg Oncol. 1971;3:539-51.

9. Martini N, Bains MS, Burt ME, Zakowski MF, McCormack P, Rusch VW, et al. Incidence of local recurrence and second primary tumors in resected Stage I lung cancer. J Thoracic Cardiovasc Surg. 1995;109:120-9.

10. Paul S, Altorki NK, Sheng S, Lee PC, Harpole DH, Onaitis MW, et al. Thoracoscopic lobectomy is associated with lower morbidity than open lobectomy: a propensity-matched analysis from the STS database. J Thorac Cardiovasc Surg. 2010;139:366-78.

11. Flores RM, Park BJ, Dycoco J, Aronova A, Hirth Y, Rizk NP, et al. Lobectomy by video-assisted thoracic surgery (VATS) versus thoracotomy for lung cancer. J Thorac Cardiovasc Surg. 2009;138:11-8.

12. Flores RM, Ihekweazu UN, Rizk N, Dycoco J, Bains MS, Downey RJ, et al. Patterns of recurrence and incidence of second primary tumors after lobectomy by VATS versus thoracotomy for lung cancer. J Thorac Cardiovasc Surg. 2011;141:59-64.

13. McKenna RJ, Houck W, Fuller CB. Video-assisted thoracic surgery lobectomy: experience with 1100 cases. Ann Thorac Surg. 2006;81:421-6.

14. Yankelevitz DF, Reeves A, Kostis W, Zhao B, Henschke CI. Determination of malignancy in small pulmonary nodules based on volumetrically determined growth rates: preliminary results. Radiology. 2000;217:251-6.

15. Kostis WJ, Yankelevitz DF, Reeves AP, Fluture SC, Henschke CI. Small pulmonary nodules: reproducibility of three-dimensional volumetric measurement and estimation of time to follow-up CT. Radiology. 2004;231: 446-52.

16. Liu X, Liang M, Wang Y, Chen K, Chen X, Qin P, et al. The outcome differences of CT screening for lung cancer: pre and post following an algorithm in Zhuhai, China. Lung Cancer. 2011;73:230-6.

17. Wagnetz U, Menezes RJ, Boerner S, Paul NS, Wagnetz D, Keshavjee S, et al. CT screening for lung cancer: implication of lung biopsy recommendations. AJR Am J Roentgenol. 2012;198:351-8.

18. Cardillo G, Regal M, Sera F, Di Martino M, Carbone L, Facciolo F, et al. Videothoracoscopic management of the solitary pulmonary nodule: a singleinstitution study on 429 cases. Ann Thorac Surg. 2003;75:1607-11.

19. DeCamp MM Jr, Jaklitsch MT, Mentzer SJ, Harpole DH, Sugarbaker DJ. The safety and versatility of video-thoracoscopy: a prospective analysis of 895 consecutive cases. J Am Coll Surg. 1995;181:113-20.
20. Bernard A, Ferrand L, Hagry O, Benoit L, Cheynel N, Favre JP. Identification of prognostic factors determining risk groups for lung resection. Ann Thorac Surg. 2000;70:1161-7.

21. Smith MA, Battafarano RJ, Meyers BF, Zoole JB, Cooper JD, Patterson GA Prevalence of benign disease in patients undergoing resection for suspected lung cancer. Ann Thorac Surg. 2006;81:1824-9.

22. Ginsberg RJ, Rubinstein LV. Randomized trial of lobectomy versus limited resection for T1 N0 non-small cell lung cancer. Lung Cancer Study Group. Ann Thorac Surg. 1995;60:615-22.

23. Starnes SL, Reed MF, Meyer CA, Shipley RT, Jazieh AR, Pina EM, et al. Can lung cancer screening by computed tomography be effective in areas with endemic histoplasmosis? J Thorac Cardiovasc Surg. 2011;141:688-93.

24. Field JK, Smith RA, Aberle DR, Oudkerk M, Baldwin DR, Yankelevitz D, et al International Association for the Study of Lung Cancer Computed Tomography Screening Workshop 2011 report. J Thorac Oncol. 2012;7:10-9.

25. Boffa DJ, Allen MS, Grab JD, Gaissert HA, Harpole DH, Wright CD. Data from the Society of Thoracic Surgeons General Thoracic Surgery database: the surgical management of primary lung tumors. J Thorac Cardiovasc Surg. 2008; 135:247-54.

26. Humphrey LL, Deffebach M, Pappas M, Baumann C, Artis K, Mitchell JP, et al. Screening for lung cancer with low-dose computed tomography: a systematic review to update the U.S. Preventive Services Task Force recommendation. Ann Intern Med. 2013;159:411-20.

27. Pastorino U, Borasio P, Francese M, Miceli R, Calabrò E, Solli P, et al Lung cancer stage is an independent risk factor for surgical mortality. Tumori. 2008;94:362-9.

28. Ginsberg RJ, Hill LD, Eagan RT, Thomas P, Mountain CF, Deslauriers J, et al Modern thirty-day operative mortality for surgical resections in lung cancer. J Thorac Cardiovasc Surg. 1983;86:654-8.

\section{APPENDIX 1}

The I-ELCAP Investigators: Mount Sinai School of Medicine, New York, NY: Claudia I. Henschke (Principal Investigator), David F. Yankelevitz, Raja Flores, Rowena Yip, Dongming Xu. Weill Cornell Medical College, New York, NY: Dorothy I. McCauley, Mildred Chen, Daniel M. Libby, James P. Smith, Mark Pasmantier, Nasser Altorki. Cornell University, Ithaca, NY: Anthony P. Reeves, Alberto Biancardi, Center for the Biology of Natural Systems, City University of New York at Queens College, Queens, NY: Steven Markowitz, Albert Miller; Christiana Care. Helen F. Graham Cancer Center, Newark, Del: Thomas Bauer. National Cancer Institute, Swedish Medical Center, Seattle, Wash: Ralph Aye. Columbia University Medical Center, New York, NY: John H.M. Austin, Belinda M. D'Souza, Gregory D.N. Pearson. St Agnes Cancer Center, Baltimore, Md: Enser Cole. New York University Medical Center, New York, NY: David Naidich, Georgeann McGuinness. Holy Cross Hospital Cancer Institute, Silver Spring, Md: Cheryl Aylesworth. State University of New York at Stony Brook, Stony Brook, NY: Matthew Rifkin. Maimonides Medical Center, Brooklyn, NY: Samuel Kopel. Roswell Park Cancer Institute, Buffalo, NY: Donald Klippenstein. State University of New York, Upstate Medical Center, Syracuse, NY: Leslie J. Kohman, Ernest M. Scalzetti. Dorothy E. Schneider Cancer Center, Mills-Peninsula Health Services, San Mateo, Calif: Barry Sheppard. ProHealth Care Regional Cancer Center, Waukesha \& Oconomowoc Memorial Hospitals, Oconomowoc, Wis: M. Kristin Thorsen, Richard Hansen. North Shore-Long Island Jewish 
Health System, New Hyde Park, NY: Arfa Khan, Rakesh Shah. Jackson Memorial Hospital, University of Miami, Miami, Fla: Richard Thurer, Tammy Baxter. Eisenhower Lucy Curci Cancer Center, Rancho Mirage, Calif; Davood Vafai. South Nassau Communities Hospital, Long Island, NY: Shahriyour Andaz. Mount Sinai School of Medicine, New York, NY: David S. Mendelson. Georgia Institute for Lung Cancer Research, Atlanta, Ga: Michael V. Smith. Nebraska Methodist Hospital, Omaha, Neb: Patrick Meyers. St. Joseph Health Center, St Charles, Mo: Dan Luedke. Memorial Sloan-Kettering Cancer Center, New York, NY: Robert T. Heelan, Michelle S. Ginsberg. New York Medical College, Valhalla, NY: Terence A.S. Matalon. Mount Sinai Comprehensive Cancer Center, Miami Beach, Fla: Shari-Lynn Odzer. Wellstar Health System, Marietta, Ga: William Mayfield. Aurora St. Luke's Medical Center, Milwaukee, Wis: David Olsen. City of Hope National Medical Center, Duarte, Calif: Fred Grannis, Arnold Rotter. St Joseph's Hospital, Atlanta, Ga: Paul
Scheinberg. Evanston Northwestern Healthcare Medical Group, Evanston, Ill: Daniel Ray; Staten Island University Hospital, Staten Island, NY: Mary Salvatore. Our Lady of Mercy Medical Center, Bronx, NY: Peter H. Wiernik. The Valley Hospital Cancer Center, Paramus, NJ: Robert Korst. Greenwich Hospital, Greenwich, Conn: David Mullen. Glens Falls Hospital, Glens Falls, NY: Louis DeCunzo. Karmanos Cancer Institute, Detroit, Mich: Harvey Pass, Carmen Endress. Baylor University Medical Center, Dallas, Tex: Ed Cheung. Sharp Memorial Hospital, San Diego, Calif: Michael Kalafer. John Muir Cancer Institute, Concord, Calif: Michaela Straznicka. Comprehensive Cancer Center, Sequoia Hospital, Redwood City, Calif: Melissa Lim. Alta Bates Summit Medical Center, Berkeley, Calif: Gary Cecchi. Rush University Medical Center, Chicago, Ill: Mark Yoder. Beth Israel Hospital Center, New York, NY: Cliff Connery. Comprehensive Cancer Center, Bend Memorial Hospital, Bend, Ore: Albert Koch. 